\title{
Compact and continuous embeddings of logarithmic Bessel potential spaces
}

\author{
by \\ David E. Edmunds (Brighton), Petr Gurka (Praha) \\ and Bohumír OpIC (Praha)
}

\begin{abstract}
We establish compact and continuous embeddings for Bessel potential spaces modelled upon generalized Lorentz-Zygmund spaces. The target spaces are either of Lorentz-Zygmund or Hölder type.
\end{abstract}

1. Introduction. In a series of recent papers [EGO1-6] a systematic investigation of embeddings of Bessel potential spaces modelled upon generalized Lorentz-Zygmund (GLZ) spaces was carried out. (Bessel potential spaces of this kind are often called logarithmic Bessel potential spaces.) For a survey of our results we refer to $[\mathrm{O}]$. In $[\mathrm{N}]$ some of these results were extended to the case when GLZ spaces are replaced by Lorentz-Karamata spaces.

Let $p, q \in(0, \infty], m, n \in \mathbb{N}, \alpha_{1}, \ldots, \alpha_{m} \in \mathbb{R}$ and let $\Omega$ be a domain in $\mathbb{R}^{n}$. Then $L_{p, q ; \alpha_{1}, \ldots, \alpha_{m}}(\Omega)$ is the GLZ space defined to be the set of all functions $f$ on $\Omega$ such that

$$
\left\|t^{1 / p-1 / q}\left(\prod_{j=1}^{m} \ell_{j}^{\alpha_{j}}(t)\right) f^{*}(t)\right\|_{L^{q}(0, \infty)}<\infty .
$$

Here $f^{*}$ stands for the non-increasing rearrangement of $f$ and

$$
\text { (1.1) } \quad \ell_{1}(t):=1+|\log t|, \quad \ell_{j}(t):=\ell_{1}\left(\ell_{j-1}\right)(t) \quad(j>1), \quad t \in(0, \infty)
$$

are the logarithmic functions. These spaces contain many familiar objects including Lebesgue, Lorentz, Zygmund and Lorentz-Zygmund spaces. Corresponding to each space $X=X\left(\mathbb{R}^{n}\right)=L_{p, q ; \alpha_{1}, \ldots, \alpha_{m}}\left(\mathbb{R}^{n}\right)$ with $p \in(1, \infty)$,

2000 Mathematics Subject Classification: 46E35, 46E30, 26D10.

Key words and phrases: Bessel potential spaces, spaces of Hölder-continuous functions, Lorentz-Zygmund spaces, continuous embeddings, compact embeddings.

The research was partially supported by grant no. 201/01/0333 of the Grant Agency of the Czech Republic, by NATO grant PST. CLG. 978694, and by Leverhulme Trust grant $\mathrm{F} / 00407 / \mathrm{E}$. 
$q \in[1, \infty]$ and to each $\sigma>0$, the logarithmic Bessel potential space $H^{\sigma} X$ is defined to be the set of all convolutions $g_{\sigma} * f$, where $g_{\sigma}$ is the usual Bessel potential kernel and $f \in X$; this space is (quasi-)normed by means of the (quasi-)norm of $X$. When $\sigma \in \mathbb{N}$ and $p, q \in(1, \infty), H^{\sigma} X$ turns out to coincide with $W^{\sigma} X$, the Sobolev space of order $\sigma$ modelled upon $X$.

Embedding theorems for $H^{\sigma} X$ obtained in [EGO1-6] extend the classical Sobolev theorems and give embeddings into GLZ spaces, Orlicz spaces of multiple exponential type or Hölder-like spaces, depending on the parameter values. Although many results were obtained, the research is not yet complete. For example, in the sublimiting situation (when $0<\sigma<n$ and $1<p<n / \sigma)$ we established sharp continuous embeddings into GLZ spaces and proved that such embeddings are not compact. On the other hand, with the classical Sobolev embeddings in mind, we might expect that these embeddings become compact if the parameters involved in the target spaces are restricted in a proper way. One of our aims is to show that this is really the case. While in the classical situation compactness is achieved by restricting the parameter on the power-type level, in our general situation we show (cf. Theorem 3.1 and Corollaries 3.2) that the same effect is caused by each shift (in an appropriate way) of any parameter of the logarithmic levels. Similar results are obtained in the limiting situation (when $0<\sigma<n$ and $p=n / \sigma)$-cf. Theorem 3.4 and Corollaries 3.5.

Another question which we answer concerns embeddings into classes of $\lambda(\cdot)$-Hölder continuous functions. Such embeddings for spaces $H^{\sigma} X\left(\mathbb{R}^{n}\right), X$ still being a GLZ space, were established in [EGO4] and their sharpness was proved in [EGO6], both provided that $\sigma>1$. The second aim of this paper is to analyse the situation when $\sigma \in(0,1]$. In such a case one cannot use the method in which a lifting argument (based on [EGO4; Lemma 4.1] which extends the Calderón result [Ca; Thm. 7]) is applied to reduce the superlimiting case to the sublimiting one. Nevertheless, we have succeeded in establishing embeddings of $H^{\sigma} X$ into $\lambda(\cdot)$-Hölder classes in the superlimiting case even when $\sigma \in(0,1]$ — cf. Section 4 .

The third goal of this paper concerns the embedding of $H^{\sigma} X\left(\mathbb{R}^{n}\right), X$ being the GLZ space $L_{p, q ; \alpha_{1}, \ldots, \alpha_{m}}$, into spaces of $\lambda(\cdot)$-Hölder continuous functions in the limiting situation when $\sigma=n / p$, the logarithmic exponents $\alpha_{j}, j=1, \ldots, m-1$, have limiting values $1 / q^{\prime}$, and $\alpha_{m}>1 / q^{\prime}$. Such embeddings are established in Theorem 4.3. As a corollary, we obtain an interesting result which has no analogue in the classical theory of embeddings of Sobolev-Orlicz spaces. Namely, Theorem 4.3 implies that the Sobolev-Orlicz space $W^{k} L^{n / k}(\log L)^{\alpha}\left(\mathbb{R}^{n}\right), k \in \mathbb{N}$ and $k<n$ (the Sobolev space modelled upon the Orlicz space $L^{n / k}(\log L)^{\alpha}\left(\mathbb{R}^{n}\right) \equiv L_{\Phi}\left(\mathbb{R}^{n}\right)$, where the Young function satisfies $\left.\Phi(t)=\left[t(1+|\log t|)^{\alpha}\right]^{n / k}, t>0\right)$, is continuously embedded 
into the $\lambda(\cdot)$-Hölder class $C^{0, \lambda(\cdot)}\left(\overline{\mathbb{R}^{n}}\right)$ with

$$
\lambda(t)=(1+|\log t|)^{-\alpha+1-k / n}, \quad t>0,
$$

provided that $\alpha>1-k / n$ (the function $\lambda(t)$ tends to 0 as $t \rightarrow 0_{+}$more slowly than any function $t^{\varepsilon}$ with $\varepsilon>0$ ). This complements Corollary 4.6 in [EGO4] and illustrates the important role of the logarithmic term $(\log L)^{\alpha}$ involved in the Sobolev-Orlicz space $W^{k} L^{n / k}(\log L)^{\alpha}\left(\mathbb{R}^{n}\right)$. (By the classical results, the Sobolev space $W^{k, n / k}\left(\mathbb{R}^{n}\right)=W^{k} L^{n / k}\left(\mathbb{R}^{n}\right), k \in \mathbb{N}$ and $k<n$, is not even continuously embedded into the space $L^{\infty}(\Omega)$ for any domain $\Omega \subset \mathbb{R}^{n}$.) If $k=1$ and $\mathbb{R}^{n}$ is replaced by a bounded strongly Lipschitz domain $\Omega \subset \mathbb{R}^{n}$, then such a result also follows from [Ci; Thm. 3.15]. (Recall that Theorem 3.15 of $[\mathrm{Ci}]$ is stronger than Theorem 8.36 of $[\mathrm{A}]$.) The embedding mentioned above (with $\lambda$ from (1.2)) should also be compared with the following corollary of [EGO4; Thm. 4.11] (which extends the result of [BW] about "almost Lipschitz continuity"): $W^{k+1} L^{n / k}(\log L)^{\alpha}\left(\mathbb{R}^{n}\right) \hookrightarrow C^{0, \lambda(\cdot)}\left(\overline{\mathbb{R}^{n}}\right), k \in \mathbb{N}$ and $k<n$, with

$$
\lambda(t)=t(1+|\log t|)^{-\alpha+1-k / n}, \quad t>0,
$$

provided that $\alpha<1-k / n$.

The paper is organized as follows. Section 2 contains the notation and auxiliary results. Compactness of sublimiting and limiting embeddings is treated in Section 3, while Section 4 is devoted to embeddings into classes of $\lambda(\cdot)$-Hölder continuous functions. Some auxiliary results of Section 2 are proved in the Appendix.

2. Notation and preliminaries. By $c, C, C_{1}, C_{2}$ etc. we denote positive constants independent of appropriate quantities. We write $A \lesssim B$ (or $A \gtrsim B)$ if $A \leq c B$ (resp. $c A \geq B$ ), and $A \approx B$ if both $A \lesssim B$ and $A \gtrsim B$. For $p \in[1, \infty]$, the conjugate number $p^{\prime}$ is defined by $1 / p+1 / p^{\prime}=1$ with the convention that $1 / \infty=0$. Throughout the paper we also adopt the convention that $1 / 0=+\infty$.

Let $\Omega$ be a measurable subset of $\mathbb{R}^{n}$ (with respect to $n$-dimensional Lebesgue measure); by $|\Omega|_{n}$ we mean its $\left(n\right.$-)volume while $\chi_{\Omega}$ stands for the characteristic function of $\Omega$. The volume and the surface area of the unit ball $B_{n}(0,1)$ in $\mathbb{R}^{n}$ are denoted by $\omega_{n}$ and by $s_{n}$, respectively. The symbol $\mathfrak{M}(\Omega)$ is used to denote the family of all scalar-valued (real or complex) measurable functions on the set $\Omega ; \mathfrak{M}^{+}(\Omega)$ stands for the subset of $\mathfrak{M}(\Omega)$ consisting of those $f$ which are non-negative a.e. in $\Omega$. If $\Omega=(a, b) \subseteq \mathbb{R}$, we write simply $\mathfrak{M}(a, b)$ and $\mathfrak{M}^{+}(a, b)$ instead of $\mathfrak{M}((a, b))$ and $\mathfrak{M}^{+}((a, b))$. The symbol $\mathfrak{M}^{+}(a, b ; \downarrow)$ stands for the collection of all $f \in \mathfrak{M}^{+}(a, b)$ which are non-increasing on $(a, b)$. Finally, by $\mathcal{W}(\Omega)$ (or by $\mathcal{W}(a, b)$ ) we mean the class of weight functions on $\Omega$ (resp. on $(a, b)$ ) consisting of all measurable functions which are positive a.e. on $\Omega$ (resp. on $(a, b)$ ). 
Embeddings. Given two quasi-Banach spaces $X$ and $Y$, we write $X=Y$ (and say that $X$ and $Y$ coincide) if $X$ and $Y$ are equal in the algebraic and the topological sense (their quasi-norms are equivalent). The symbol $X \hookrightarrow Y$ or $X \hookrightarrow \hookrightarrow Y$ means that $X \subset Y$ and the natural embedding of $X$ in $Y$ is continuous or compact, respectively.

Spaces of continuous functions. By the symbol $C_{\mathrm{B}}(\Omega)$ we denote the space of bounded continuous functions on a domain $\Omega \subset \mathbb{R}^{n}$, that is,

$$
C_{\mathrm{B}}(\Omega):=C(\Omega) \cap L^{\infty}(\Omega),
$$

equipped with the $L^{\infty}(\Omega)$ norm.

Let $\mathcal{L}$ be the class of all continuous functions $\lambda:(0, \infty) \rightarrow(0, \infty)$ which are increasing on some interval $(0, \delta)$, with $\delta=\delta(\lambda)>0$, and satisfy $\lim _{\lambda \rightarrow 0_{+}} \lambda(t)=0$. Let $\lambda \in \mathcal{L}$ and let $\Omega$ be a domain in $\mathbb{R}^{n}$. The space $C^{0, \lambda(\cdot)}(\bar{\Omega})$ of $\lambda(\cdot)$-Hölder continuous functions consists of those functions $u \in C(\bar{\Omega})$ for which the norm

$$
\|u\|_{C^{0, \lambda(\cdot)}(\bar{\Omega})}:=\sup _{x \in \Omega}|u(x)|+\sup _{\substack{x, y \in \Omega \\ x \neq y}} \frac{|u(x)-u(y)|}{\lambda(|x-y|)}
$$

is finite; here $C(\bar{\Omega})$ stands for the family of all functions which are bounded and uniformly continuous on $\Omega$. As usual, by the space of Hölder continuous functions with exponent $\alpha \in(0,1]$ we mean the space $C^{0, \alpha}(\bar{\Omega}):=C^{0, \lambda(\cdot)}(\bar{\Omega})$, where $\lambda(t)=t^{\alpha}, t>0$; when $\alpha=1$ this space is usually called the space of Lipschitz continuous functions. For more information about such spaces see [A] or [KJF].

Lorentz-type spaces. Let $p, q \in(0, \infty]$, let $\Omega$ be a domain in $\mathbb{R}^{n}$ and let $b \in \mathcal{W}\left(0,|\Omega|_{n}\right)$ be such that

$$
B_{p, q ; b}(t):=\left\|\tau^{1 / p-1 / q} b(\tau)\right\|_{q ;(0, t)}<\infty \quad \text { for all } t \in\left(0,|\Omega|_{n}\right),
$$

where $\|\cdot\|_{q ; E}$ is the usual $L^{q}$-(quasi-)norm on the measurable set $E$. The Lorentz-type space $L_{p, q ; b}(\Omega)$ consists of all functions $f \in \mathfrak{M}(\Omega)$ for which the quantity

$$
\|f\|_{p, q ; b ; \Omega}:=\left\|t^{1 / p-1 / q} b(t) f^{*}(t)\right\|_{q ;\left(0,|\Omega|_{n}\right)}
$$

is finite; here $f^{*}$ denotes the non-increasing rearrangement of $f$ given by

$$
f^{*}(t)=\inf \left\{\lambda>0 ;|\{x \in \Omega ;|f(x)|>\lambda\}|_{n} \leq t\right\}, \quad t \geq 0 .
$$

We shall also need the inequality (cf. [BS; p. 41])

$$
(f+g)^{*}(t) \leq f^{*}(t / 2)+g^{*}(t / 2), \quad t \geq 0
$$


and the maximal function $f^{* *}$ of $f^{*}$ defined by

$$
f^{* *}(t)=\frac{1}{t} \int_{0}^{t} f^{*}(s) d s, \quad t>0 .
$$

(Clearly, $f^{*} \leq f^{* *}$; we refer to [BS; Chapt. 2] for more properties of $f^{*}$ and $f^{* *}$.)

One can show that the functional (2.2) is a quasi-norm on $L_{p, q ; b}(\Omega)$ if and only if the function $B_{p, q ; b}$ given by (2.1) satisfies

$$
B_{p, q ; b} \in \Delta_{2},
$$

that is, $B_{p, q ; b}(2 t) \lesssim B_{p, q ; b}(t)$ for all $t \in\left(0,|\Omega|_{n} / 2\right)$. (This follows, e.g., from [CS; Cor. 2] if $q \in(0, \infty)$. When $q=\infty$, then arguments similar to those used in the proof of [CS; Cor. 2] together with inequality (2.4) and the fact that

$$
\|f\|_{p, \infty ; b ; \Omega}=\left\|B_{p, \infty ; b}(t) f^{*}(t)\right\|_{\infty,\left(0,|\Omega|_{n}\right)} \quad \text { for all } f \in L_{p, \infty ; b}(\Omega)
$$

yield the result. Note also that equality (2.6) follows on interchanging the essential suprema involved on its right-hand side.)

In particular, one can easily verify that (2.5) is satisfied provided that

$$
b(2 t) \lesssim b(t) \quad \text { for a.e. } t \in\left(0,|\Omega|_{n} / 2\right) .
$$

Moreover, since the relation $b \in \mathcal{W}\left(0,|\Omega|_{n}\right)$ yields $B_{p, q ; b}(t)>0$ for all $t \in$ $\left(0,|\Omega|_{n}\right)$, one can prove that the space $L_{p, q ; b}(\Omega)$ is complete (cf. the proof of Proposition 2.2.9 in [CRS]; if $q=\infty$ one makes use of (2.6) again).

If $q \in[1, \infty)$, the spaces $L_{p, q ; b}(\Omega)$ are particular cases of the classical Lorentz spaces $\Lambda^{q}(w)$ introduced by Lorentz [L] (see also [DL]). On the other hand, if $b$ is a slowly varying function (cf. [GOT]), then $L_{p, q ; b}(\Omega)$ is the Lorentz-Karamata space. In particular, if

$$
b(t)=\prod_{j=1}^{m} \ell_{j}^{\alpha_{j}}(t), \quad t>0,
$$

where $m \in \mathbb{N}, \alpha_{1}, \ldots, \alpha_{m} \in \mathbb{R}$ and the logarithmic functions are defined on $(0, \infty)$ by $(1.1)$, then the space $L_{p, q ; b}(\Omega)$ coincides with the generalized Lorentz-Zygmund (GLZ) space $L_{p, q ; \alpha_{1}, \ldots, \alpha_{m}}(\Omega)$ from [EGO4]. Let us note that when each $\alpha_{j}=0$, the space $L_{p, q ; \alpha_{1}, \ldots, \alpha_{m}}(\Omega)$ is the Lorentz space $L^{p, q}(\Omega)$, which is just the Lebesgue space $L^{p}(\Omega)$ when $p=q$. If $m=1$, then $L_{p, q ; \alpha_{1}}(\Omega)$ is the Lorentz-Zygmund space $L^{p, q}(\log L)^{\alpha_{1}}(\Omega)$ introduced in [BR] which, when $p=q$, is the Zygmund class $L^{p}(\log L)^{\alpha_{1}}(\Omega)$. For more information about GLZ spaces $L_{p, q ; \alpha_{1}, \ldots, \alpha_{m}}(\Omega)$ see [EGO1-6] and [OP].

If $\Omega=\mathbb{R}^{n}$, we sometimes omit this symbol in the notation and, for example, write $\|\cdot\|_{p, q ; \alpha_{1}, \ldots, \alpha_{m}}$ or $L_{p, q ; \alpha_{1}, \ldots, \alpha_{m}}$ instead of $\|\cdot\|_{p, q ; \alpha_{1}, \ldots, \alpha_{m} ; \mathbb{R}^{n}}$ or $L_{p, q ; \alpha_{1}, \ldots, \alpha_{m}}\left(\mathbb{R}^{n}\right)$, respectively. 
Convergence in measure. Let $\Omega$ be a measurable subset of $\mathbb{R}^{n}$. We say that the sequence $\left\{u_{i}\right\}_{i=1}^{\infty}$ of functions converges in measure to a measurable function $u$ on $\Omega$ (notation $u_{i} \stackrel{\text { meas }}{\longrightarrow} u$ on $\Omega$ ) if, for every $\varepsilon>0$,

$$
\lim _{i \rightarrow \infty}\left|\left\{x \in \Omega ;\left|u_{i}(x)-u(x)\right| \geq \varepsilon\right\}\right|_{n}=0 .
$$

Uniform absolute continuity. Let $Y=Y(\Omega)$ be a Lorentz-type space. We say that a subset $K$ of $Y$ has uniformly absolutely continuous norm in the space $Y$, written $K \subset \operatorname{UAC}(Y)$, if for every $\varepsilon>0$ there exists $\delta>0$ such that

$$
\left\|u \chi_{M}\right\|_{Y} \leq \varepsilon \quad \text { for all } u \in K \text { and every } M \subset \Omega \text { with }|M|_{n}<\delta .
$$

We shall need the following two assertions.

Lemma 2.1. Let $\Omega$ be a domain in $\mathbb{R}^{n}$. Suppose that a sequence $\left\{u_{i}\right\}_{i} \subset$ $L^{1}(\Omega)$ converges to $u$ in $L^{1}(\Omega)$. Then $u_{i} \stackrel{\text { meas }}{\longrightarrow} u$ on $\Omega$.

For the proof see [A; proof of Theorem 8.23].

Lemma 2.2. Let $K \subset \operatorname{UAC}(Y)$, where $Y=L_{p, q ; b}(\Omega)$ is a Lorentz-type space such that $B_{p, q ; b} \in \Delta_{2}$ and $\left\|\chi_{\Omega}\right\|_{Y} \equiv B_{p, q ; b}\left(|\Omega|_{n}\right)<\infty$. Then every sequence $\left\{u_{i}\right\}_{i} \subset K$ which converges in measure on $\Omega$ converges also in the quasi-norm of $Y$.

Proof. Take $\varepsilon>0$ and $\left\{u_{i}\right\}_{i} \subset K$. Since $K \subset \operatorname{UAC}(Y)$, there is $\delta>0$ such that (2.8) holds with $u$ replaced by $u_{i}$ (for all $i$ ). Assume that $u_{i} \stackrel{\text { meas }}{\longrightarrow} u$ on $\Omega$ and put

$$
\Omega_{i j}(\varepsilon)=\left\{x \in \Omega ;\left|u_{i}(x)-u_{j}(x)\right| \geq \varepsilon\right\}, \quad i, j \in \mathbb{N} .
$$

Since $\Omega_{i j}(\varepsilon) \subseteq \Omega_{i}(\varepsilon / 2) \cup \Omega_{j}(\varepsilon / 2)$, where

$$
\Omega_{k}(\varepsilon / 2)=\left\{x \in \Omega ;\left|u_{k}(x)-u(x)\right| \geq \varepsilon / 2\right\}, \quad k \in \mathbb{N},
$$

the assumption $u_{i} \stackrel{\text { meas }}{\longrightarrow} u$ on $\Omega$ implies that there is $N \in \mathbb{N}$ such that $\left|\Omega_{i j}(\varepsilon)\right|_{n}<\delta$ for every $i, j \in \mathbb{N}, i, j>N$. Thus, using also the lattice property of $Y$ and the inequality $\left\|\chi_{\Omega}\right\|_{Y}<\infty$, we obtain

$$
\begin{aligned}
\left\|u_{i}-u_{j}\right\|_{Y} & \lesssim\left\|\left(u_{i}-u_{j}\right) \chi_{\Omega_{i j}(\varepsilon)}\right\|_{Y}+\left\|\left(u_{i}-u_{j}\right) \chi_{\Omega \backslash \Omega_{i j}(\varepsilon)}\right\|_{Y} \\
& \lesssim\left\|u_{i} \chi_{\Omega_{i j}(\varepsilon)}\right\|_{Y}+\left\|u_{j} \chi_{\Omega_{i j}(\varepsilon)}\right\|_{Y}+\left\|\left(u_{i}-u_{j}\right) \chi_{\Omega \backslash \Omega_{i j}(\varepsilon)}\right\|_{Y} \\
& \lesssim \varepsilon+\varepsilon+\varepsilon\left\|\chi_{\Omega}\right\|_{Y} \lesssim \varepsilon .
\end{aligned}
$$

Consequently, $\left\{u_{i}\right\}_{i}$ is a Cauchy sequence in $Y$ and the result follows since $Y$ is complete.

Logarithmic Bessel potential spaces. The Bessel kernel $g_{\sigma}, \sigma>0$, is defined to be the function on $\mathbb{R}^{n}$ whose Fourier transform $\widehat{g}_{\sigma}$ is

$$
\widehat{g}_{\sigma}(\xi)=(2 \pi)^{-n / 2}\left(1+|\xi|^{2}\right)^{-\sigma / 2}, \quad \xi \in \mathbb{R}^{n},
$$


where by the Fourier transform $\widehat{f}$ of a function $f$ we mean

$$
\widehat{f}(x)=(2 \pi)^{-n / 2} \int_{\mathbb{R}^{n}} e^{-i x \cdot y} f(y) d y, \quad x \in \mathbb{R}^{n} .
$$

Let $\sigma>0, p \in(1, \infty), q \in[1, \infty], \alpha_{1}, \ldots, \alpha_{m} \in \mathbb{R}$. The logarithmic Bessel potential space $H^{\sigma} L_{p, q ; \alpha_{1}, \ldots, \alpha_{m}}\left(\mathbb{R}^{n}\right)$ is defined by

$$
H^{\sigma} L_{p, q ; \alpha_{1}, \ldots, \alpha_{m}}\left(\mathbb{R}^{n}\right):=\left\{u=g_{\sigma} * f ; f \in L_{p, q ; \alpha_{1}, \ldots, \alpha_{m}}\left(\mathbb{R}^{n}\right)\right\},
$$

and is equipped with the (quasi-)norm

$$
\|u\|_{\sigma ; p, q ; \alpha_{1}, \ldots, \alpha_{m}}:=\|f\|_{p, q ; \alpha_{1}, \ldots, \alpha_{m}} .
$$

(By $f * g$ we mean the convolution of functions $f$ and $g$.) If $\Omega \subset \mathbb{R}^{n}$ is a domain, then

$$
H^{\sigma} L_{p, q ; \alpha_{1}, \ldots, \alpha_{m}}(\Omega):=\left\{\left.\widetilde{u}\right|_{\Omega} ; \widetilde{u} \in H^{\sigma} L_{p, q ; \alpha_{1}, \ldots, \alpha_{m}}\left(\mathbb{R}^{n}\right)\right\}
$$

and this space is endowed with the (quasi-)norm

$$
\|u\|_{\sigma ; p, q ; \alpha_{1}, \ldots, \alpha_{m} ; \Omega}:=\inf \left\{\|\widetilde{u}\|_{\sigma ; p, q ; \alpha_{1}, \ldots, \alpha_{m}} ; u=\left.\widetilde{u}\right|_{\Omega}\right\} .
$$

Properties of the Bessel kernel. Let us summarize the basic properties of the Bessel kernel $g_{\sigma}$ :

(2.11) $g_{\sigma}$ is a positive, integrable function which is analytic except at the origin;

(2.12) $\quad g_{\sigma}(x) \leq C_{1}|x|^{\sigma-n} e^{-C_{2}|x|}$ for $0<\sigma<n$ and all $x \in \mathbb{R}^{n}$;

(2.13) $\quad g_{\sigma}(x) \approx|x|^{\sigma-n}$ as $|x| \rightarrow 0$ if $0<\sigma<n$;

(2.14) $\left|\frac{\partial}{\partial x_{j}} g_{\sigma}(x)\right| \leq C|x|^{\sigma-n-1}$ for $0<\sigma \leq n+1, j \in\{1, \ldots, n\}, x \in$ $\mathbb{R}^{n} \backslash\{0\}$

$$
\begin{aligned}
& g_{\sigma}^{*}(t) \lesssim t^{(\sigma-n) / n} e^{-C t^{1 / n}} \text { for } 0<\sigma<n \text { and all } t>0 \\
& g_{\sigma}^{* *}(t) \lesssim\left\{\begin{array}{ll}
t^{(\sigma-n) / n} & \text { for all } t \in(0,1) \\
t^{-1} & \text { for all } t>1
\end{array} \text { and } 0<\sigma<n\right.
\end{aligned}
$$

For the proof of (2.11)-(2.14) see [AMS], for (2.15) and (2.16) see [EGO2].

O'Neil's inequality. Let $f, g \in \mathfrak{M}\left(\mathbb{R}^{n}\right)$. Then

$$
(f * g)^{*}(t) \leq(f * g)^{* *}(t) \leq t f^{* *}(t) g^{* *}(t)+\int_{t}^{\infty} f^{*}(\tau) g^{*}(\tau) d \tau, \quad t>0 .
$$

For the proof we refer to [O'N] or $[\mathrm{Z}]$.

Together with (2.15) and (2.16), this immediately yields the following result. 
Corollary 2.3. Let $0<\sigma<n$. Then there is a positive constant $c$ such that, for all $f \in \mathfrak{M}\left(\mathbb{R}^{n}\right)$,

$\left(g_{\sigma} * f\right)^{*}(t) \leq c\left(t^{(\sigma-n) / n} \int_{0}^{t} f^{*}(\tau) d \tau+\int_{t}^{\infty} f^{*}(\tau) \tau^{(\sigma-n) / n} e^{-C \tau^{1 / n}} d \tau\right)$ for all $t \in(0,1]$,

and

$$
\left(g_{\sigma} * f\right)^{*}(t) \leq c\left(f^{* *}(t)+\int_{t}^{\infty} f^{*}(\tau) \tau^{(\sigma-n) / n} e^{-C \tau^{1 / n}} d \tau\right) \quad \text { for all } t \in(1, \infty) .
$$

Hardy inequalities. Let $1 \leq q \leq \infty,-\infty \leq a<b \leq \infty$ and let $w_{1}, w_{2}, v \in$ $\mathcal{W}(a, b)$.

(i) The inequality

$$
\left\|w_{1}(t) \int_{a}^{t} w_{2}(\tau) \varphi(\tau) d \tau\right\|_{q ;(a, b)} \lesssim \mathcal{B}_{1}(a, b)\|v \varphi\|_{q ;(a, b)}
$$

holds for all $\varphi \in \mathfrak{M}^{+}(a, b)$ if and only if

$$
\mathcal{B}_{1}(a, b):=\sup _{a<R<b}\left\|w_{1}\right\|_{q ;(R, b)}\left\|w_{2} v^{-1}\right\|_{q^{\prime} ;(a, R)}<\infty .
$$

(ii) The inequality

$$
\left\|w_{1}(t) \int_{t}^{b} w_{2}(\tau) \varphi(\tau) d \tau\right\|_{q ;(a, b)} \lesssim \mathcal{B}_{2}(a, b)\|v \varphi\|_{q ;(a, b)}
$$

holds for all $\varphi \in \mathfrak{M}^{+}(a, b)$ if and only if

$$
\mathcal{B}_{2}(a, b):=\sup _{a<R<b}\left\|w_{1}\right\|_{q ;(a, R)}\left\|w_{2} v^{-1}\right\|_{q^{\prime} ;(R, b)}<\infty .
$$

For the proof we refer to [OK; pp. 13, 55 and 63].

We shall make use of the following lemma whose proof can be found in the Appendix.

Lemma 2.4. Let $0<\sigma<n, 1<p<\infty, q \in[1, \infty], m \in \mathbb{N}$ and $\alpha_{1}, \ldots, \alpha_{m} \in \mathbb{R}$. Let $\Omega$ be a bounded domain in $\mathbb{R}^{n}$. Then

$$
\left.H^{\sigma} L_{p, q ; \alpha_{1}, \ldots, \alpha_{m}}\left(\mathbb{R}^{n}\right) \hookrightarrow \hookrightarrow L^{1}(\Omega) .{ }^{1}\right)
$$

Difference operator. For each $h \in \mathbb{R}^{n}$, the first difference operator $\Delta_{h}$ is defined on functions on $\mathbb{R}^{n}$ by

$$
\Delta_{h} f(x)=f(x+h)-f(x), \quad x \in \mathbb{R}^{n} .
$$

$\left({ }^{1}\right)$ This means that the mapping $\left.u \mapsto u\right|_{\Omega}$ from $H^{\sigma} L_{p, q ; \alpha_{1}, \ldots, \alpha_{m}}\left(\mathbb{R}^{n}\right)$ into $L^{1}(\Omega)$ is compact. 
We shall need the following two estimates involving the first difference of $g_{\sigma}$. For their proofs see the Appendix.

Lemma 2.5. Let $0<\sigma<n, \max \{1, n / \sigma\}<p$, and $p<n /(\sigma-1)$ if $\sigma>1$. Assume that $1<q<\infty, m \in \mathbb{N}$ and $\alpha_{1}, \ldots, \alpha_{m} \in \mathbb{R}$. Then, for all $h \in \mathbb{R}^{n}$ with $|h|>0$,

$$
\left\|\Delta_{h} g_{\sigma}\right\|_{p^{\prime}, q^{\prime} ;-\alpha_{1}, \ldots,-\alpha_{m}} \lesssim|h|^{\sigma-n / p} \prod_{j=1}^{m} \ell_{j}^{-\alpha_{j}}(|h|) .
$$

Lemma 2.6. Let $0<\sigma<n$ and $p=n / \sigma$. Assume that $1<q<\infty$, $m \in \mathbb{N}$,

$$
\alpha_{m}>1 / q^{\prime} \text { and, if } m>1 \text {, let } \alpha_{j}=1 / q^{\prime} \text { for } j=1, \ldots, m-1 .
$$

Then, for all $h \in \mathbb{R}^{n}$ with $|h|>0$,

$$
\left\|\Delta_{h} g_{\sigma}\right\|_{p^{\prime}, q^{\prime} ;-\alpha_{1}, \ldots,-\alpha_{m}} \lesssim \ell_{m}^{1 / q^{\prime}-\alpha_{m}}(|h|) .
$$

\section{Compact embeddings into Lorentz-type spaces}

Theorem 3.1. Let $0<\sigma<n, 1<p<n / \sigma, 1 / p_{\sigma}=1 / p-\sigma / n$, $q \in[1, \infty], m \in \mathbb{N}$ and $\alpha_{1}, \ldots, \alpha_{m} \in \mathbb{R}$. Let $\Omega$ be a bounded domain in $\mathbb{R}^{n}$. Assume that $b \in \mathcal{W}\left(0,|\Omega|_{n}\right)$ is such that $B_{p_{\sigma}, q ; b} \in \Delta_{2}, B_{p_{\sigma}, q ; b}\left(|\Omega|_{n}\right)<\infty$ and

$$
\lim _{t \rightarrow 0_{+}} \frac{b(t)}{\prod_{j=1}^{m} \ell_{j}^{\alpha_{j}}(t)}=0 .
$$

Then

$$
H^{\sigma} L_{p, q ; \alpha_{1}, \ldots, \alpha_{m}}\left(\mathbb{R}^{n}\right) \hookrightarrow \hookrightarrow L_{p_{\sigma}, q ; b}(\Omega) .
$$

Proof. By [EGO4; Thm. 4.8],

$$
H^{\sigma} L_{p, q ; \alpha_{1}, \ldots, \alpha_{m}}\left(\mathbb{R}^{n}\right) \hookrightarrow L_{p_{\sigma}, q ; \alpha_{1}, \ldots, \alpha_{m}}(\Omega) .
$$

Put

$$
K=\left\{u \in H^{\sigma} L_{p, q ; \alpha_{1}, \ldots, \alpha_{m}} ;\|u\|_{\sigma ; p, q ; \alpha_{1}, \ldots, \alpha_{m}} \leq 1\right\} .
$$

If $\left\{u_{i}^{\prime}\right\}_{i} \subset K$, then Lemma 2.4 implies that there is a subsequence $\left\{u_{i}\right\}_{i} \subset$ $\left\{u_{i}^{\prime}\right\}_{i}$ such that $u_{i} \rightarrow u$ in $L^{1}(\Omega)$. Thus, by Lemma 2.1, $\left\{u_{i}\right\}_{i}$ converges to $u$ in measure on $\Omega$. In view of Lemma 2.2, it is sufficient to show that $K \subset \operatorname{UAC}\left(L_{p_{\sigma}, q ; b}(\Omega)\right)$.

Let $\varepsilon>0$. By (3.1), there is $\delta \in\left(0,|\Omega|_{n}\right)$ such that

$$
b(t) \leq \varepsilon \prod_{j=1}^{m} \ell_{j}^{\alpha_{j}}(t) \quad \text { for all } t \in(0, \delta) .
$$


Assume that $u \in K$ and let $M \subset \Omega$ satisfy $|M|_{n}<\delta$. Since $u=g_{\sigma} * f$, where $\|f\|_{p, q ; \alpha_{1}, \ldots, \alpha_{m}} \leq 1$ (cf. (2.9), (2.10)) and

$$
\left(u \chi_{M}\right)^{*} \leq u^{*} \chi_{(0, \delta)}
$$

we deduce from (3.3) and (3.2) that

$$
\begin{aligned}
\left\|u \chi_{M}\right\|_{p_{\sigma}, q ; b ; \Omega} & \leq\left\|t^{1 / p_{\sigma}-1 / q} b(t) u^{*}(t)\right\|_{q ;(0, \delta)} \\
& \leq \varepsilon\left\|t^{1 / p_{\sigma}-1 / q}\left(\prod_{j=1}^{m} \ell_{j}^{\alpha_{j}}(t)\right) u^{*}(t)\right\|_{q ;(0, \delta)} \\
& \lesssim \varepsilon\|f\|_{p, q ; \alpha_{1}, \ldots, \alpha_{m}} \leq \varepsilon
\end{aligned}
$$

and the result follows.

Corollaries 3.2. Let $0<\sigma<n, 1<p<n / \sigma, 1 / p_{\sigma}=1 / p-\sigma / n$, $q \in[1, \infty], m \in \mathbb{N}$ and $\alpha_{1}, \ldots, \alpha_{m} \in \mathbb{R}$. Let $\Omega$ be a bounded domain in $\mathbb{R}^{n}$.

(i) Assume that $\beta_{j} \in \mathbb{R}^{n}, j \in\{1, \ldots, m\}$, satisfy

$$
\begin{array}{cl}
\text { either } & \beta_{1}<\alpha_{1} \\
\text { or } & \beta_{1}=\alpha_{1}, \beta_{2}<\alpha_{2} \\
\vdots & \\
\text { or } & \beta_{1}=\alpha_{1}, \beta_{2}=\alpha_{2}, \ldots, \beta_{m-1}=\alpha_{m-1}, \beta_{m}<\alpha_{m} .
\end{array}
$$

Then

$$
H^{\sigma} L_{p, q ; \alpha_{1}, \ldots, \alpha_{m}}\left(\mathbb{R}^{n}\right) \hookrightarrow \hookrightarrow L_{p_{\sigma}, q ; \beta_{1}, \ldots, \beta_{m}}(\Omega) .
$$

(ii) Assume that $0<\bar{p}<p_{\sigma}$ and $\beta_{j} \in \mathbb{R}^{n}, j \in\{1, \ldots, m\}$. Then

$$
H^{\sigma} L_{p, q ; \alpha_{1}, \ldots, \alpha_{m}}\left(\mathbb{R}^{n}\right) \hookrightarrow \hookrightarrow L_{\bar{p}, q ; \beta_{1}, \ldots, \beta_{m}}(\Omega) .
$$

Proof. For $t>0$, put

$$
b(t)= \begin{cases}\prod_{j=1}^{m} \ell_{j}^{\beta_{j}}(t) & \text { in case }(\mathrm{i}), \\ t^{1 / \bar{p}-1 / p_{\sigma}} \prod_{j=1}^{m} \ell_{j}^{\beta_{j}}(t) & \text { in case }(\mathrm{ii}) .\end{cases}
$$

Then the result follows from Theorem 3.1.

Remark 3.3. The results of Theorem 3.1 and Corollary 3.2(i) are optimal. This follows from the fact that the embedding

$$
H^{\sigma} L_{p, q ; \alpha_{1}, \ldots, \alpha_{m}}\left(\mathbb{R}^{n}\right) \hookrightarrow L_{p_{\sigma}, q ; \alpha_{1}, \ldots, \alpha_{m}}(\Omega) .
$$

is continuous (and sharp) but not compact (cf. [EGO6; Thm. 3.1]).

Theorem 3.4. Let $0<\sigma<n, p=n / \sigma, q \in[1, \infty], m \in \mathbb{N}, \alpha_{m}<$ $1 / q^{\prime}, \beta_{m}=\alpha_{m}-1$ and, if $m>1$, let $\alpha_{1}=\cdots=\alpha_{m-1}=1 / q^{\prime}$ and 
$\beta_{1}=\cdots=\beta_{m-1}=-1 / q$. Let $\Omega$ be a bounded domain in $\mathbb{R}^{n}$. Assume that $b \in \mathcal{W}\left(0,|\Omega|_{n}\right)$ is such that $B_{\infty, q ; b} \in \Delta_{2}, B_{\infty, q ; b}\left(|\Omega|_{n}\right)<\infty$ and

$$
\lim _{t \rightarrow 0_{+}} \frac{b(t)}{\prod_{j=1}^{m} \ell_{j}^{\beta_{j}}(t)}=0 .
$$

Then

$$
H^{\sigma} L_{p, q ; \alpha_{1}, \ldots, \alpha_{m}}\left(\mathbb{R}^{n}\right) \hookrightarrow \hookrightarrow L_{\infty, q ; b}(\Omega) .
$$

Proof. Put $X=L_{p, q ; \alpha_{1}, \ldots, \alpha_{m}}\left(\mathbb{R}^{n}\right)$. As in the proof of Theorem 3.1, it is sufficient to verify that

$$
K:=\left\{u \in H^{\sigma} X ;\|u\|_{H^{\sigma} X} \leq 1\right\} \subset \operatorname{UAC}\left(L_{\infty, q ; b}(\Omega)\right) .
$$

Let $\varepsilon>0$. By (3.5), there is $\delta \in\left(0, \min \left\{1,|\Omega|_{n}\right\}\right)$ such that

$$
b(t) \leq \varepsilon \prod_{j=1}^{m} \ell_{j}^{\beta_{j}}(t) \quad \text { for all } t \in(0, \delta) .
$$

Assume that $u \in K$ and let $M \subset \Omega$ satisfy $|M|_{n}<\delta$. From (3.4) and (3.6) we obtain

$$
\left\|u \chi_{M}\right\|_{\infty, q ; b ; \Omega} \leq \varepsilon\left\|t^{-1 / q}\left(\prod_{j=1}^{m} \ell_{j}^{\beta_{j}}(t)\right) u^{*}(t)\right\|_{q ;(0, \delta)} .
$$

Consequently, since $u=g_{\sigma} * f$ with $\|f\|_{X} \leq 1$, it is enough to prove that

$$
\left\|t^{-1 / q}\left(\prod_{j=1}^{m} \ell_{j}^{\beta_{j}}(t)\right) u^{*}(t)\right\|_{q ;(0, \delta)} \lesssim\|f\|_{X} \quad \text { for all } f \in X .
$$

Such an estimate is a consequence of [N; Cor. 5.2], where a more general result is proved in the context of Bessel potential spaces modelled upon Lorentz-Karamata spaces.

For the convenience of the reader, we give a proof of (3.8) here. By Corollary 2.3,

$$
\left\|t^{-1 / q}\left(\prod_{j=1}^{m} \ell_{j}^{\beta_{j}}(t)\right) u^{*}(t)\right\|_{q ;(0, \delta)} \lesssim \mathcal{J}_{1}+\mathcal{J}_{2}+\mathcal{J}_{3},
$$

where

$$
\begin{aligned}
& \mathcal{J}_{1}:=\left\|t^{-1 / q}\left(\prod_{j=1}^{m} \ell_{j}^{\beta_{j}}(t)\right) t^{(\sigma-n) / n} \int_{0}^{t} f^{*}(\tau) d \tau\right\|_{q ;(0, \delta)}, \\
& \mathcal{J}_{2}:=\left\|t^{-1 / q}\left(\prod_{j=1}^{m} \ell_{j}^{\beta_{j}}(t)\right) \int_{t}^{\delta} f^{*}(\tau) \tau^{(\sigma-n) / n} d \tau\right\|_{q ;(0, \delta)},
\end{aligned}
$$




$$
\mathcal{J}_{3}:=\left\|t^{-1 / q}\left(\prod_{j=1}^{m} \ell_{j}^{\beta_{j}}(t)\right) \int_{\delta}^{\infty} f^{*}(\tau) \tau^{(\sigma-n) / n} e^{-C \tau^{1 / n}} d \tau\right\|_{q ;(0, \delta)} .
$$

To estimate $\mathcal{J}_{1}$, we use the Hardy inequality (2.17):

$$
\begin{aligned}
\mathcal{J}_{1} & =\left\|t^{-1 / p^{\prime}-1 / q}\left(\prod_{j=1}^{m} \ell_{j}^{\beta_{j}}(t)\right) \int_{0}^{t} f^{*}(\tau) d \tau\right\|_{q ;(0, \delta)} \\
& \lesssim \mathcal{D}_{1}(\delta)\left\|t^{1 / p-1 / q}\left(\prod_{j=1}^{m} \ell_{j}^{\alpha_{j}}(t)\right) f^{*}(t)\right\|_{q ;(0, \delta)}
\end{aligned}
$$

where

$$
\mathcal{D}_{1}(\delta):=\sup _{0<R<\delta}\left\|t^{-1 / p^{\prime}-1 / q} \prod_{j=1}^{m} \ell_{j}^{\beta_{j}}(t)\right\|_{q ;(R, \delta)}\left\|\left(t^{1 / p-1 / q} \prod_{j=1}^{m} \ell_{j}^{\alpha_{j}}(t)\right)^{-1}\right\|_{q^{\prime} ;(0, R)} .
$$

Since, for all $R \in(0, \delta)$,

$$
\left\|t^{-1 / p^{\prime}-1 / q} \prod_{j=1}^{m} \ell_{j}^{\beta_{j}}(t)\right\|_{q ;(R, \delta)} \lesssim R^{-1 / p^{\prime}} \prod_{j=1}^{m} \ell_{j}^{\beta_{j}}(R)
$$

and

$$
\begin{aligned}
\left\|\left(t^{1 / p-1 / q} \prod_{j=1}^{m} \ell_{j}^{\alpha_{j}}(t)\right)^{-1}\right\|_{q^{\prime} ;(0, R)} & =\left\|t^{1 / p^{\prime}-1 / q^{\prime}} \prod_{j=1}^{m} \ell_{j}^{-\alpha_{j}}(t)\right\|_{q^{\prime} ;(0, R)} \\
& \lesssim R^{1 / p^{\prime}} \prod_{j=1}^{m} \ell_{j}^{-\alpha_{j}}(R)
\end{aligned}
$$

we obtain

$$
\mathcal{D}_{1}(\delta) \lesssim \sup _{0<R<\delta} \prod_{j=1}^{m} \ell_{j}^{\beta_{j}-\alpha_{j}}(R)=\sup _{0<R<\delta} \prod_{j=1}^{m} \ell_{j}^{-1}(R) \leq 1,
$$

which implies that

$$
\mathcal{J}_{1} \lesssim\|f\|_{X}
$$

To estimate $\mathcal{J}_{2}$, we apply the Hardy inequality (2.18):

$$
\begin{aligned}
\mathcal{J}_{2} & =\left\|t^{-1 / q}\left(\prod_{j=1}^{m} \ell_{j}^{\beta_{j}}(t)\right) \int_{t}^{\delta} f^{*}(\tau) \tau^{-1 / p^{\prime}} d \tau\right\|_{q ;(0, \delta)} \\
& \lesssim \mathcal{D}_{2}(\delta)\left\|t^{1 / p-1 / q}\left(\prod_{j=1}^{m} \ell_{j}^{\alpha_{j}}(t)\right) f^{*}(t)\right\|_{q ;(0, \delta)},
\end{aligned}
$$


where

$$
\begin{aligned}
\mathcal{D}_{2}(\delta):= & \sup _{0<R<\delta}\left\|t^{-1 / q} \prod_{j=1}^{m} \ell_{j}^{\beta_{j}}(t)\right\|_{q ;(0, R)} \\
& \times\left\|t^{-1 / p^{\prime}}\left(t^{1 / p-1 / q} \prod_{j=1}^{m} \ell_{j}^{\alpha_{j}}(t)\right)^{-1}\right\|_{q^{\prime} ;(R, \delta)} .
\end{aligned}
$$

Since, for all $R \in(0, \delta]$,

$$
\begin{aligned}
\left\|t^{-1 / q} \prod_{j=1}^{m} \ell_{j}^{\beta_{j}}(t)\right\|_{q ;(0, R)} & =\left\|t^{-1 / q}\left(\prod_{j=1}^{m-1} \ell_{j}^{-1 / q}(t)\right) \ell_{m}^{\alpha_{m}-1}(t)\right\|_{q ;(0, R)} \\
& \approx \ell_{m}^{\alpha_{m}-1 / q^{\prime}}(R)
\end{aligned}
$$

and

$$
\begin{aligned}
& \left\|t^{-1 / p^{\prime}}\left(t^{1 / p-1 / q} \prod_{j=1}^{m} \ell_{j}^{\alpha_{j}}(t)\right)^{-1}\right\|_{q^{\prime} ;(R, \delta)} \\
& \quad=\left\|t^{-1 / q^{\prime}}\left(\prod_{j=1}^{m-1} \ell_{j}^{-1 / q^{\prime}}(t)\right) \ell_{m}^{-\alpha_{m}}(t)\right\|_{q^{\prime} ;(R, \delta)} \lesssim \ell_{m}^{-\alpha_{m}+1 / q^{\prime}}(R),
\end{aligned}
$$

we arrive at

$$
\mathcal{J}_{2} \lesssim\|f\|_{X}
$$

Now, we are going to derive an upper estimate of $\mathcal{J}_{3}$. Since

$$
\begin{aligned}
V(\delta) & :=\left\|\tau^{-1 / q^{\prime}}\left(\prod_{j=1}^{m} \ell_{j}^{-\alpha_{j}}(\tau)\right) e^{-C \tau^{1 / n}}\right\|_{q^{\prime} ;(\delta, \infty)} \\
& \lesssim\left\|\tau^{-1 / q^{\prime}} \prod_{j=1}^{m} \ell_{j}^{-\alpha_{j}}(\tau)\right\|_{q^{\prime} ;(\delta, 1)}+1 \\
& =\left\|\tau^{-1 / q^{\prime}}\left(\prod_{j=1}^{m-1} \ell_{j}^{-1 / q^{\prime}}(\tau)\right) \ell_{m}^{-\alpha_{m}}(\tau)\right\|_{q^{\prime} ;(\delta, 1)}+1 \approx \ell_{m}^{-\alpha_{m}+1 / q^{\prime}}(\delta),
\end{aligned}
$$

the Hölder inequality yields

$$
\int_{\delta}^{\infty} f^{*}(\tau) \tau^{-1+1 / p} e^{-C \tau^{1 / n}} d \tau \leq V(\delta)\|f\|_{X} \lesssim \ell_{m}^{-\alpha_{m}+1 / q^{\prime}}(\delta)\|f\|_{X}
$$

Together with (3.11), this implies that

$$
\mathcal{J}_{3} \lesssim \ell_{m}^{-\alpha_{m}+1 / q^{\prime}}(\delta)\|f\|_{X}\left\|t^{-1 / q} \prod_{j=1}^{m} \ell_{j}^{\beta_{j}}(t)\right\|_{q ;(0, \delta)} \approx\|f\|_{X}
$$

Combining estimates (3.9), (3.10), (3.12) and (3.13), we arrive at (3.8). 
Corollaries 3.5. Let $0<\sigma<n, q \in[1, \infty], m \in \mathbb{N}, \alpha_{m}<1 / q^{\prime}$, $\beta_{m}=\alpha_{m}-1$ and, if $m>1$, let $\alpha_{1}=\cdots=\alpha_{m-1}=1 / q^{\prime}$ and $\beta_{1}=\cdots=$ $\beta_{m-1}=-1 / q$. Let $\Omega$ be a bounded domain in $\mathbb{R}^{n}$.

(i) Assume that $\gamma_{j} \in \mathbb{R}, j \in\{1, \ldots, m\}$, satisfy

$$
\begin{aligned}
\text { either } & \gamma_{1}<\beta_{1}, \\
\text { or } & \gamma_{1}=\beta_{1}, \gamma_{2}<\beta_{2}, \\
& \vdots \\
\text { or } & \gamma_{1}=\beta_{1}, \gamma_{2}=\beta_{2}, \ldots, \gamma_{m-1}=\beta_{m-1}, \gamma_{m}<\beta_{m} .
\end{aligned}
$$

Then

$$
H^{\sigma} L_{n / \sigma, q ; \alpha_{1}, \ldots, \alpha_{m}}\left(\mathbb{R}^{n}\right) \hookrightarrow \hookrightarrow L_{\infty, q ; \gamma_{1}, \ldots, \gamma_{m}}(\Omega) .
$$

(ii) Assume that $\alpha<\alpha_{m}-1 / q^{\prime}$. Then

$$
H^{\sigma} L_{n / \sigma, q ; \alpha_{1}, \ldots, \alpha_{m}}\left(\mathbb{R}^{n}\right) \hookrightarrow \hookrightarrow \operatorname{EXP}_{m} L^{-1 / \alpha}(\Omega)
$$

where $\operatorname{EXP}_{m} L^{-1 / \alpha}(\Omega):=L_{\Phi}(\Omega)$ and $L_{\Phi}$ is the Orlicz space with the Young function $\Phi$ given by

$$
\Phi(t)=(\underbrace{\exp \circ \cdots \circ \exp }_{m \text { times }})\left(t^{-1 / \alpha}\right) \quad \text { for all large } t>0 .
$$

(iii) Assume that $0<\bar{p}<\infty$ and $\gamma_{j} \in \mathbb{R}$ for $j \in\{1, \ldots, m\}$. Then

$$
H^{\sigma} L_{n / \sigma, q ; \alpha_{1}, \ldots, \alpha_{m}}\left(\mathbb{R}^{n}\right) \hookrightarrow \hookrightarrow L_{\bar{p}, q ; \gamma_{1}, \ldots, \gamma_{m}}(\Omega) .
$$

Proof. For $t>0$, put

$$
b(t)= \begin{cases}\prod_{j=1}^{m} \ell_{j}^{\gamma_{j}}(t) & \text { in case }(\mathrm{i}), \\ t^{1 / \bar{p}} \prod_{j=1}^{m} \ell_{j}^{\gamma_{j}}(t) & \text { in case (iii). }\end{cases}
$$

Then the assertions of parts (i) and (iii) follow from Theorem 3.4.

The result of part (ii) is a consequence of that given in part (i). Indeed, since $\operatorname{EXP}_{m} L^{-1 / \alpha}(\Omega)=L_{\infty, \infty ; \ell_{m}^{\alpha}}(\Omega)$ (cf. [OP; Lemma 8.1]), it is sufficient to show that, for any $q \in[1, \infty]$,

$$
L_{\infty, q ; \gamma_{1}, \ldots, \gamma_{m}}(\Omega) \hookrightarrow L_{\infty, \infty ; \ell_{m}^{\alpha}}(\Omega),
$$

where $\gamma_{1}=\cdots=\gamma_{m-1}=-1 / q$ and $\gamma_{m}=\alpha-1 / q$. As $f^{*} \in \mathfrak{M}^{+}(0, \infty ; \downarrow)$ and

$$
\ell_{m}^{\alpha}(t) \approx\left\|\tau^{-1 / q} \prod_{j=1}^{m} \ell_{j}^{\gamma_{j}}(\tau)\right\|_{q ;(0, t)} \quad \text { for all } t \in\left(0,|\Omega|_{n}\right)
$$


we obtain

$$
\begin{aligned}
\|f\|_{\infty, \infty ; \ell_{m}^{\alpha} ; \Omega} & =\operatorname{essup}_{0<t<|\Omega|_{n}} \ell_{m}^{\alpha}(t) f^{*}(t) \\
& \lesssim \operatorname{essiup}_{0<t<|\Omega|_{n}}\left\|\tau^{-1 / q}\left(\prod_{j=1}^{m} \ell_{j}^{\gamma_{j}}(\tau)\right) f^{*}(\tau)\right\|_{q ;(0, t)} \\
& =\|f\|_{\infty, q ; \gamma_{1}, \ldots, \gamma_{m} ; \Omega},
\end{aligned}
$$

which yields (3.15).

REMARKS 3.6. Let $0<\sigma<n, q \in[1, \infty], m \in \mathbb{N}, \alpha_{m}<1 / q^{\prime}$ and, if $m>1$, let $\alpha_{1}=\cdots=\alpha_{m-1}=1 / q^{\prime}$. Let $\Omega$ be a bounded domain in $\mathbb{R}^{n}$.

(i) Since the embedding

$$
H^{\sigma} L_{n / \sigma, q ; \alpha_{1}, \ldots, \alpha_{m}}\left(\mathbb{R}^{n}\right) \hookrightarrow \operatorname{EXP}_{m} L^{-1 /\left(\alpha_{m}-1 / q^{\prime}\right)}(\Omega)
$$

fails to be compact (cf. [EGO3]), we cannot replace $\alpha$ in (3.14) with its limiting value $\alpha_{m}-1 / q^{\prime}$.

(ii) The same method as that used to derive (3.8) yields the embedding

$$
H^{\sigma} L_{n / \sigma, q ; \alpha_{1}, \ldots, \alpha_{m}}\left(\mathbb{R}^{n}\right) \hookrightarrow L_{\infty, q ; \beta_{1}, \ldots, \beta_{m}}(\Omega)
$$

with $\beta_{1}, \ldots, \beta_{m}$ from Theorem 3.4. One can again see that (3.17) is not compact. (Indeed, assuming compactness of (3.17) and combining this embedding with the continuous embedding (cf. (3.15))

$$
L_{\infty, q ; \beta_{1}, \ldots, \beta_{m}}(\Omega) \hookrightarrow L_{\infty, \infty ; \ell_{m}^{\alpha_{m}-1 / q^{\prime}}}(\Omega)=\operatorname{EXP}_{m} L^{-1 /\left(\alpha_{m}-1 / q^{\prime}\right)}(\Omega),
$$

we obtain compactness of (3.16), which is a contradiction.)

4. Embeddings into $\lambda(\cdot)$-Hölder continuous functions. The following assertion is an extension of [EGO4; Thm. 4.9], where the case $1 \leq$ $\sigma<n+1$ was treated by a quite different method.

Theorem 4.1. Let $0<\sigma<n, n / \sigma<p<\infty$, and $p<n /(\sigma-1)$ if $\sigma>1$. Assume that $1<q<\infty, m \in \mathbb{N}, \alpha_{1}, \ldots, \alpha_{m} \in \mathbb{R}$ and put

$$
\lambda(t)=t^{\sigma-n / p} \prod_{j=1}^{m} \ell_{j}^{-\alpha_{j}}(t), \quad t>0 .
$$

Then

$$
H^{\sigma} L_{p, q ; \alpha_{1}, \ldots, \alpha_{m}}\left(\mathbb{R}^{n}\right) \hookrightarrow C^{0, \lambda(\cdot)}\left(\overline{\mathbb{R}^{n}}\right) .
$$

Proof. Let $p, q, \alpha_{1}, \ldots, \alpha_{m}$ satisfy the assumptions of Theorem 4.1. If $X=L_{p, q ; \alpha_{1}, \ldots, \alpha_{m}}\left(\mathbb{R}^{n}\right)$, then its associate space $X^{\prime}$ is given by $X^{\prime}=$ $L_{p^{\prime}, q^{\prime} ;-\alpha_{1}, \ldots,-\alpha_{m}}\left(\mathbb{R}^{n}\right)$ (cf. [EGO4; Lemma 3.4]). 
Let $u \in H^{\sigma} X$ with $\|u\|_{H^{\sigma} X} \leq 1$. Then there is a function $f \in X$ such that $u=g_{\sigma} * f$ and $\|f\|_{X}=\|u\|_{H^{\sigma} X} \leq 1$. Therefore

$$
\begin{aligned}
|u(x+h)-u(x)| & =\left|\int_{\mathbb{R}^{n}} f(y)\left[g_{\sigma}(x+h-y)-g_{\sigma}(x-y)\right] d y\right| \\
& \leq\|f\|_{X}\left\|\Delta_{h} g_{\sigma}\right\|_{X^{\prime}} \lesssim\left\|\Delta_{h} g_{\sigma}\right\|_{X^{\prime}} .
\end{aligned}
$$

Together with Lemma 2.5, this yields

$|u(x+h)-u(x)| \lesssim \lambda(|h|) \quad$ for all $x \in \mathbb{R}^{n}$ and $h \in \mathbb{R}^{n}$ with $|h|>0$.

Since also $H^{\sigma} X \hookrightarrow C_{\mathrm{B}}\left(\mathbb{R}^{n}\right)$ by [EGO4; Cor. 4.6], the result follows.

Combining Theorem 4.1 with [EGO4; Thm. 4.9], we arrive at the following result.

TheOREm 4.2. Let $0<\sigma<n+1, \max \{1, n / \sigma\}<p<\infty$, and $p<$ $n /(\sigma-1)$ if $\sigma>1$. Assume that $1<q<\infty, m \in \mathbb{N}, \alpha_{1}, \ldots, \alpha_{m} \in \mathbb{R}$ and put

Then

$$
\lambda(t)=t^{\sigma-n / p} \prod_{j=1}^{m} \ell_{j}^{-\alpha_{j}}(t), \quad t>0 .
$$

$$
H^{\sigma} L_{p, q ; \alpha_{1}, \ldots, \alpha_{m}}\left(\mathbb{R}^{n}\right) \hookrightarrow C^{0, \lambda(\cdot)}\left(\overline{\mathbb{R}^{n}}\right) .
$$

The next assertion is a complement of [EGO4; Cor. 4.6] and gives the embedding mentioned in the Introduction (cf. (1.2)).

Theorem 4.3. Let $0<\sigma<n, p=n / \sigma, 1<q<\infty, m \in \mathbb{N}, \alpha_{m}>1 / q^{\prime}$ and, if $m>1$, let $\alpha_{j}=1 / q^{\prime}$ for $j=1, \ldots, m-1$. Put

$$
\lambda(t)=\ell_{m}^{1 / q^{\prime}-\alpha_{m}}(t), \quad t>0
$$

Then

$$
H^{\sigma} L_{p, q ; \alpha_{1}, \ldots, \alpha_{m}}\left(\mathbb{R}^{n}\right) \hookrightarrow C^{0, \lambda(\cdot)}\left(\overline{\mathbb{R}^{n}}\right) .
$$

The proof is analogous to that of Theorem 4.1 (instead of Lemma 2.5 one makes use of Lemma 2.6).

CoRollary 4.4. Let the assumptions of Theorem 4.2 or Theorem 4.3, respectively, be satisfied. Assume that $\Omega \subset \mathbb{R}^{n}$ is a bounded domain and that $\lambda_{1} \in \mathcal{L}$ is such that $\lambda / \lambda_{1} \in \mathcal{L}$. Then

$$
H^{\sigma} L_{p, q ; \alpha_{1}, \ldots, \alpha_{m}}\left(\mathbb{R}^{n}\right) \hookrightarrow \hookrightarrow C^{0, \lambda_{1}(\cdot)}\left(\overline{\mathbb{R}^{n}}\right) .
$$

Proof. Corollary 4.4 is a consequence of Theorem 4.2 or Theorem 4.3, respectively, and [EGO4; Lemma 4.15(iv)].

REMARK 4.5. Note that if the condition $\alpha_{m}>1 / q^{\prime}$ in Theorem 4.3 is replaced by $\alpha_{m}<1 / q^{\prime}$, then (instead of (4.1))

$$
H^{\sigma} L_{p, q ; \alpha_{1}, \ldots, \alpha_{m}}\left(\mathbb{R}^{n}\right) \hookrightarrow \operatorname{EXP}_{m} L^{-1 /\left(\alpha_{m}-1 / q^{\prime}\right)}(\Omega)
$$

for any bounded domain $\Omega$ in $\mathbb{R}^{n}$ (cf. Remarks 3.6). 


\section{Appendix}

Proof of Lemma 2.4. Put $X=X\left(\mathbb{R}^{n}\right)=L_{p, q ; b}\left(\mathbb{R}^{n}\right)$, where $b(t)=$ $\prod_{j=1}^{m} \ell_{j}^{\alpha_{j}}(t), t>0$. Let $B$ be an open ball in $\mathbb{R}^{n}$ containing $\Omega$. Take $\delta \in(0, \min \{\sigma, n / p\})$ and $\bar{p} \in\left(p, p_{\delta}\right)$, where $1 / p_{\delta}=1 / p-\delta / n$. Then the desired embedding is a consequence of the following chain of embeddings:

$$
H^{\sigma} X\left(\mathbb{R}^{n}\right) \hookrightarrow H^{\sigma-\delta} L^{\bar{p}}\left(\mathbb{R}^{n}\right) \hookrightarrow \hookrightarrow L^{\bar{p}}(B) \hookrightarrow L^{1}(\Omega) .
$$

The last embedding in (5.1) is obvious. For the second one observe that if $\sigma-\delta=k \in \mathbb{N}$, then the fact that (see [S; Thm. 3 of Chapt. V]) $H^{k} L^{\bar{p}}\left(\mathbb{R}^{n}\right)=W^{k, \bar{p}}\left(\mathbb{R}^{n}\right)$, where $W^{k, \bar{p}}\left(\mathbb{R}^{n}\right)$ is a Sobolev space, the obvious embedding $W^{k, \bar{p}}\left(\mathbb{R}^{n}\right) \hookrightarrow W^{k, \bar{p}}(B)$ and the compact embedding $W^{k, \bar{p}}(B) \hookrightarrow \hookrightarrow$ $L^{\bar{p}}(B)$ (see [EE; Thm. V.4.17]) imply that

$$
H^{k} L^{\bar{p}}\left(\mathbb{R}^{n}\right) \hookrightarrow \hookrightarrow L^{\bar{p}}(B),
$$

which is the second embedding in (5.1). If $\sigma-\delta \notin \mathbb{N}$ take $k \in \mathbb{N}$ such that $k>\sigma-\delta$. Then (5.2) holds. Moreover,

$$
L^{\bar{p}}\left(\mathbb{R}^{n}\right) \hookrightarrow L^{\bar{p}}(B) .
$$

The space $H^{\sigma-\delta} L^{\bar{p}}\left(\mathbb{R}^{n}\right)$ can be obtained by complex interpolation from $H^{k} L^{\bar{p}}\left(\mathbb{R}^{n}\right)$ and $L^{\bar{p}}\left(\mathbb{R}^{n}\right)$ (see [T; (11) of Sect. 2.4.2]). Thus, the second embedding in (5.1) is now a consequence of (5.2), (5.3) and the preservation of compactness under complex interpolation ([T; Thm. 1 of Sect. 1.16.4]). The first embedding in (5.1) follows from the fact that $g_{\sigma}=g_{\sigma-\delta} * g_{\delta}$ (cf., e.g., [Z; p. 65]) and

$$
H^{\delta} X\left(\mathbb{R}^{n}\right) \hookrightarrow L^{\bar{p}}\left(\mathbb{R}^{n}\right) .
$$

We prove (5.4), which is equivalent to the validity of the inequality

$$
\left\|u^{*}\right\|_{\bar{p} ;(0, \infty)} \lesssim\|f\|_{X} \quad \text { for all } f \in X, \text { where } u=g_{\delta} * f .
$$

We can easily see that

$$
\begin{aligned}
\left\|u^{*}\right\|_{\bar{p} ;(0,1)} & \leq\left\|t^{1 / p_{\delta}} b(t) u^{*}(t)\right\|_{\infty ;(0,1)}\left\|t^{-1 / p_{\delta}}[b(t)]^{-1}\right\|_{\bar{p} ;(0,1)} \\
& \approx\left\|t^{1 / p_{\delta}} b(t) u^{*}(t)\right\|_{\infty ;(0,1)} \quad \text { for all } u \in \mathfrak{M}\left(\mathbb{R}^{n}\right) .
\end{aligned}
$$

Let $P \in(0, \infty)$ and $Q \in(0, \infty]$. Then, for all $u \in \mathfrak{M}\left(\mathbb{R}^{n}\right)$ and every $t>0$,

$$
t^{1 / P} b(t) u^{*}(t) \approx\left\|\tau^{1 / P-1 / Q} b(\tau)\right\|_{Q ;(0, t)} u^{*}(t) \leq\left\|\tau^{1 / P-1 / Q} b(\tau) u^{*}(\tau)\right\|_{Q ;(0, t)},
$$

which implies that

$$
\left\|\tau^{1 / P} b(\tau) u^{*}(\tau)\right\|_{\infty ;(0,1)} \lesssim\left\|\tau^{1 / P-1 / Q} b(\tau) u^{*}(\tau)\right\|_{Q ;(0,1)}
$$

and

$$
u^{*}(t) \lesssim\left[t^{1 / P} b(t)\right]^{-1}\|u\|_{P, Q ; b ;(0, \infty)} .
$$

Using (5.6) and (5.7) (with $P=p_{\delta}$ and $Q=q$ ), we arrive at

$$
\left\|u^{*}\right\|_{\bar{p} ;(0,1)} \lesssim\left\|u^{*}\right\|_{p_{\delta}, q ; \alpha_{1}, \ldots \alpha_{m}} \quad \text { for all } u \in \mathfrak{M}\left(\mathbb{R}^{n}\right) .
$$


Since, by [EGO4; Thm. 4.8],

$$
H^{\delta} X\left(\mathbb{R}^{n}\right) \hookrightarrow L_{p_{\delta}, q ; \alpha_{1}, \ldots, \alpha_{m}}\left(\mathbb{R}^{n}\right),
$$

we deduce from (5.9) that

$$
\left\|u^{*}\right\|_{\bar{p} ;(0,1)} \lesssim\|f\|_{X} \quad \text { for all } f \in X \text { and } u=g_{\delta} * f .
$$

Applying Corollary 2.3 and the triangle inequality, we deduce, for all $f \in X$ and $u=g_{\delta} * f$, that

$$
\begin{aligned}
\left\|u^{*}\right\|_{\bar{p} ;(1, \infty)} & \lesssim\left\|f^{* *}\right\|_{\bar{p} ;(1, \infty)}+\left\|\int_{t}^{\infty} f^{*}(\tau) \tau^{(\delta-n) / n} e^{-C \tau^{1 / n}} d \tau\right\|_{\bar{p} ;(1, \infty)} \\
& =: N_{1}+N_{2} .
\end{aligned}
$$

By the definition of $f^{* *}$ and by (5.8) (with $P=p, Q=q, u=f$ and $t=\tau$ ),

$$
\begin{aligned}
N_{1} & \lesssim\left\|t^{-1} \int_{0}^{t} \tau^{-1 / p}[b(\tau)]^{-1} d \tau\right\|_{\bar{p} ;(1, \infty)}\|f\|_{X} \\
& \approx\left\|t^{-1 / p}[b(t)]^{-1}\right\|_{\bar{p} ;(1, \infty)}\|f\|_{X} \\
& \approx\|f\|_{X} \quad \text { for all } f \in X
\end{aligned}
$$

Finally, using (5.8) (again with $P=p, Q=q, u=f$ and $t=\tau$ ), we arrive at

$$
\begin{aligned}
\int_{t}^{\infty} f^{*}(\tau) \tau^{(\delta-n) / n} e^{-C \tau^{1 / n}} d \tau & \lesssim\|f\|_{X} \int_{t}^{\infty} \tau^{-1 / p}[b(\tau)]^{-1} \tau^{(\delta-n) / n} e^{-C \tau^{1 / n}} d \tau \\
& \lesssim\|f\|_{X} e^{-\left(C t^{1 / n}\right) / 2}
\end{aligned}
$$

which implies that

$$
N_{2} \lesssim\|f\|_{X}\left\|e^{-C t^{1 / n} / 2}\right\|_{\bar{p} ;(1, \infty)} \approx\|f\|_{X} \quad \text { for all } f \in X .
$$

Combining estimates (5.11)-(5.13), we obtain

$$
\left\|u^{*}\right\|_{\bar{p} ;(1, \infty)} \lesssim\|f\|_{X} \quad \text { for all } f \in X .
$$

Estimate (5.5) follows from (5.10) and (5.14).

Proof of Lemma 2.5. Put

$$
B(r)=\left\{x \in \mathbb{R}^{n} ;|x|<r\right\}, \quad B^{\mathrm{c}}(r)=\mathbb{R}^{n} \backslash B(r) \quad \text { for } r>0 .
$$

Let $h \in \mathbb{R}^{n}$ with $|h|>0$. Since

$$
\left\|\left(\Delta_{h} g_{\sigma}\right) \chi_{B(2|h|)}\right\|_{p^{\prime}, q^{\prime} ;-\alpha_{1}, \ldots,-\alpha_{m}} \lesssim 2\left\|g_{\sigma} \chi_{B(3|h|)}\right\|_{p^{\prime}, q^{\prime} ;-\alpha_{1}, \ldots,-\alpha_{m}},
$$

using (2.15) and the fact that $\sigma / n-1 / p>0$, we obtain 
$(5.15)$

$$
\begin{aligned}
& \left\|\left(\Delta_{h} g_{\sigma}\right) \chi_{B(2|h|)}\right\|_{p^{\prime}, q^{\prime} ;-\alpha_{1}, \ldots,-\alpha_{m}} \\
\lesssim & \left\|t^{1 / p^{\prime}-1 / q^{\prime}}\left(\prod_{j=1}^{m} \ell_{j}^{-\alpha_{j}}(t)\right) t^{\sigma / n-1}\right\|_{q^{\prime} ;\left(0, \omega_{n}(3|h|)^{n}\right)} \\
= & \left\|t^{\sigma / n-1 / p-1 / q^{\prime}} \prod_{j=1}^{m} \ell_{j}^{-\alpha_{j}}(t)\right\|_{q^{\prime} ;\left(0, \omega_{n}(3|h|)^{n}\right)} \approx|h|^{\sigma-n / p} \prod_{j=1}^{m} \ell_{j}^{-\alpha_{j}}(|h|) .
\end{aligned}
$$

Obviously

$$
\left|\Delta_{h} g_{\sigma}(x)\right| \leq|h| \sum_{j=1}^{n} \int_{0}^{1}\left|\frac{\partial}{\partial x_{j}} g_{\sigma}(x+\tau h)\right| d \tau \quad \text { for all } x \in \mathbb{R}^{n} .
$$

Moreover, if $\tau \in[0,1]$ and $x \in B^{\mathrm{c}}(2|h|)$, then

$$
\frac{1}{2}|x| \leq|x+\tau h| \leq \frac{3}{2}|x| .
$$

Consequently, (2.14) and (5.16) imply that

(5.17) $\left|\Delta_{h} g_{\sigma}(x) \chi_{B^{\mathrm{c}}(2|h|)}(x)\right| \lesssim|h||x|^{\sigma-n-1} \chi_{B^{\mathrm{c}}(|h|)}(x) \quad$ for all $x \in \mathbb{R}^{n}$.

Putting $F(x)=|x|^{\sigma-n-1} \chi_{B^{\mathrm{c}}(|h|)}(x), x \in \mathbb{R}^{n}$, and taking into account that $\sigma-n-1<0$, we can easily see that

$$
F^{*}(t)=\left(|h|^{n}+t / \omega_{n}\right)^{(\sigma-n-1) / n} \quad \text { for all } t>0 .
$$

This and (5.17) yield

$$
\begin{aligned}
& \left\|\left(\Delta_{h} g_{\sigma}\right) \chi_{B^{\mathrm{c}}(2|h|)}\right\|_{p^{\prime}, q^{\prime} ;-\alpha_{1}, \ldots,-\alpha_{m}} \\
& \quad \lesssim|h|\left\|t^{1 / p^{\prime}-1 / q^{\prime}}\left(\prod_{j=1}^{m} \ell_{j}^{-\alpha_{j}}(t)\right) F^{*}(t)\right\|_{q^{\prime} ;(0, \infty)}=|h|\left(N_{1}+N_{2}\right),
\end{aligned}
$$

where

$$
\begin{aligned}
& N_{1}:=\left\|t^{1 / p^{\prime}-1 / q^{\prime}}\left(\prod_{j=1}^{m} \ell_{j}^{-\alpha_{j}}(t)\right) F^{*}(t)\right\|_{q^{\prime} ;\left(0, \omega_{n}\left(2^{n}-1\right)|h|^{n}\right)^{\prime}}, \\
& N_{2}:=\left\|t^{1 / p^{\prime}-1 / q^{\prime}}\left(\prod_{j=1}^{m} \ell_{j}^{-\alpha_{j}}(t)\right) F^{*}(t)\right\|_{q^{\prime} ;\left(\omega_{n}\left(2^{n}-1\right)|h|^{n}, \infty\right)} .
\end{aligned}
$$

Since $\sigma-n-1<0,(5.18)$ shows that $F^{*}(t) \leq|h|^{\sigma-n-1}$ for all $t>0$. Consequently,

$$
\begin{aligned}
N_{1} & \leq|h|^{\sigma-n-1}\left\|t^{1 / p^{\prime}-1 / q^{\prime}} \prod_{j=1}^{m} \ell_{j}^{-\alpha_{j}}(t)\right\|_{q^{\prime} ;\left(0, \omega_{n}\left(2^{n}-1\right)|h|^{n}\right)} \\
& \approx|h|^{\sigma-n-1}|h|^{n / p^{\prime}} \prod_{j=1}^{m} \ell_{j}^{-\alpha_{j}}(|h|)=|h|^{\sigma-1-n / p} \prod_{j=1}^{m} \ell_{j}^{-\alpha_{j}}(|h|) .
\end{aligned}
$$


Using (5.18) and the change of variables $t=\omega_{n}\left(z^{n}-|h|^{n}\right)$, we obtain

$$
\begin{aligned}
N_{2}= & \|\left(\omega_{n}\left(z^{n}-|h|^{n}\right)\right)^{1 / p^{\prime}-1 / q^{\prime}} \\
& \times\left(\prod_{j=1}^{m} \ell_{j}^{-\alpha_{j}}\left(\omega_{n}\left(z^{n}-|h|^{n}\right)\right)\right) z^{\sigma-n-1}\left(n \omega_{n} z^{n-1}\right)^{1 / q^{\prime}} \|_{q^{\prime} ;(2|h|, \infty)} .
\end{aligned}
$$

Since $\omega_{n}\left(1-2^{-n}\right) z^{n} \leq \omega_{n}\left(z^{n}-|h|^{n}\right) \leq \omega_{n} z^{n}$ if $z>2|h|$, and $\sigma-1-n / p<0$, we arrive at

$$
\begin{aligned}
N_{2} & \approx\left\|z^{n\left(1 / p^{\prime}-1 / q^{\prime}\right)}\left(\prod_{j=1}^{m} \ell_{j}^{-\alpha_{j}}(z)\right) z^{\sigma-n-1} z^{(n-1) / q^{\prime}}\right\|_{q^{\prime} ;(2|h|, \infty)} \\
& =\left\|z^{\sigma-1-n / p-1 / q^{\prime}} \prod_{j=1}^{m} \ell_{j}^{-\alpha_{j}}(z)\right\|_{q^{\prime} ;(2|h|, \infty)} \\
& \approx|h|^{\sigma-1-n / p} \prod_{j=1}^{m} \ell_{j}^{-\alpha_{j}}(|h|)
\end{aligned}
$$

Estimates (5.19)-(5.21) imply that

$$
\left\|\left(\Delta_{h} g_{\sigma}\right) \chi_{B^{\mathrm{c}}(2|h|)}\right\|_{p^{\prime}, q^{\prime} ;-\alpha_{1}, \ldots,-\alpha_{m}} \lesssim|h|^{\sigma-n / p} \prod_{j=1}^{m} \ell_{j}^{-\alpha_{j}}(|h|) .
$$

Together with (5.15), this yields the result.

Proof of Lemma 2.6. We proceed as in the proof of Lemma 2.5. Thus, using the identity $\sigma / n-1 / p=0$ and assumption (2.19), we obtain, instead of $(5.15)$,

$$
\begin{aligned}
& \left\|\left(\Delta_{h} g_{\sigma}\right) \chi_{B(2|h|)}\right\|_{p^{\prime}, q^{\prime} ;-\alpha_{1}, \ldots,-\alpha_{m}} \\
& =\left\|t^{-1 / q^{\prime}} \prod_{j=1}^{m} \ell_{j}^{-\alpha_{j}}(t)\right\|_{q^{\prime} ;\left(0, \omega_{n}(3|h|)^{n}\right)} \approx \ell_{m}^{1 / q^{\prime}-\alpha_{m}}(|h|) .
\end{aligned}
$$

Since the estimates of the quantities $N_{1}$ and $N_{2}$ remain true, we again have (5.22), that is,

$$
\left\|\left(\Delta_{h} g_{\sigma}\right) \chi_{B^{\mathrm{c}}(2|h|)}\right\|_{p^{\prime}, q^{\prime} ;-\alpha_{1}, \ldots,-\alpha_{m}} \lesssim \prod_{j=1}^{m} \ell_{j}^{-\alpha_{j}}(|h|) .
$$

Moreover, assumption (2.19) implies that $\prod_{j=1}^{m} \ell_{j}^{-\alpha_{j}}(|h|) \leq \ell_{m}^{1 / q^{\prime}-\alpha_{m}}(|h|)$. Consequently, the assertion follows from (5.23) and (5.24).

\section{References}

[A] R. A. Adams, Sobolev Spaces, Academic Press, New York, 1975. 
[AMS] N. Aronszajn, F. Mulla and P. Szeptycki, On spaces of potentials connected with $L^{p}$ classes, Ann. Inst. Fourier (Grenoble) 13 (1963), no. 2, 211-306.

[BR] C. Bennett and K. Rudnick, On Lorentz-Zygmund spaces, Dissertationes Math. 175 (1980).

[BS] C. Bennett and R. Sharpley, Interpolation of Operators, Academic Press, Boston, 1988.

[BW] H. Brézis and S. Wainger, A note on limiting cases of Sobolev embeddings and convolution inequalities, Comm. Partial Differential Equations 5 (1980), 773789.

[Ca] A. P. Calderón, Lebesgue spaces of differentiable functions and distributions, in: Partial Differential Equations, Proc. Sympos. Pure Math. 4, Amer. Math. Soc., Providence, RI, 1961, 33-49.

[CRS] M. J. Carro, J. A. Raposo and J. Soria, Recent developments in the theory of Lorentz spaces and weighted inequalities, Mem. Amer. Math. Soc., to appear.

[CS] M. J. Carro and J. Soria, Weighted Lorentz spaces and the Hardy operator, J. Funct. Anal. 112 (1993), 480-494.

[Ci] A. Cianchi, Some results in the theory of Orlicz spaces and applications to variational problems, in: Nonlinear Analysis, Function Spaces and Applications, Vol. 6 (Prague, 1998), M. Krbec and A. Kufner (eds.), Math. Inst. Acad. Sci. Czech Republic, Prague, 1999, 50-92.

[DL] R. A. DeVore and G. G. Lorentz, Constructive Approximation, Springer, Berlin, 1993.

[EE] D. E. Edmunds and W. D. Evans, Spectral Theory and Differential Operators, Oxford Univ. Press, Oxford, 1987.

[EGO1] D. E. Edmunds, P. Gurka and B. Opic, Double exponential integrability of convolution operators in generalized Lorentz-Zygmund spaces, Indiana Univ. Math. J. 44 (1995), 19-43.

[EGO2] - - 一, Double exponential integrability, Bessel potentials and embedding theorems, Studia Math. 115 (1995), 151-181.

[EGO3] - - - - Sharpness of embeddings in logarithmic Bessel-potential spaces, Proc. Roy. Soc. Edinburgh Sect. A 126 (1996), 995-1009.

[EGO4] -, - - -, On embeddings of logarithmic Bessel potential spaces, J. Funct. Anal. 146 (1997), 116-150.

[EGO5] - - - - Norms of embeddings of logarithmic Bessel potential spaces, Proc. Amer. Math. Soc. 126 (1998), 2417-2425.

[EGO6] - - - - Optimality of embeddings of logarithmic Bessel potential spaces, Quart. J. Math. Oxford Ser. (2) 51 (2000), 185-209.

[GOT] A. Gogatishvili, B. Opic and W. Trebels, Limiting reiteration for real interpolation with slowly varying functions, Math. Nachr. 278 (2005), 1-22.

[KJF] A. Kufner, O. John and S. Fučík, Function Spaces, Academia, Prague, 1977.

[L] G. G. Lorentz, On the theory of spaces A, Pacific J. Math. 1 (1951), 411-429.

$[\mathrm{N}] \quad$ J. S. Neves, Lorentz-Karamata spaces, Bessel and Riesz potentials and embeddings, Dissertationes Math. 405 (2002).

[O'N] R. O'Neil, Convolution operators and L( $p, q)$ spaces, Duke Math. J. 30 (1963), 129-142.

[O] B. Opic, Embeddings of Bessel potential and Sobolev type spaces, in: Colloquium del Departamento de Análisis Matemático, Sección 1, Número 48, Universidad Complutense de Madrid, CURSO 1999-2000, 100-118.

[OK] B. Opic and A. Kufner, Hardy-Type Inequalities, Pitman Res. Notes in Math. Ser. 219, Longman Sci. \& Tech., Harlow, 1990. 
[OP] B. Opic and L. Pick, On generalized Lorentz-Zygmund spaces, Math. Inequal. Appl. 2 (1999), 391-467.

[S] E. M. Stein, Singular Integrals and Differentiability Properties of Functions, Princeton Univ. Press, Princeton, 1970.

[T] H. Triebel, Interpolation Theory, Function Spaces, Differential Operators, North-Holland, Amsterdam, 1978.

[Z] W. Ziemer, Weakly Differentiable Functions, Grad. Texts in Math. 120, Springer, New York, 1989.

Department of Mathematics

Mantell building

University of Sussex

Falmer, Brighton BN1 9RF, England

E-mail: d.e.edmunds@sussex.ac.uk
Department of Mathematics Czech University of Agriculture 16521 Praha 6, Czech Republic

E-mail: gurka@tf.czu.cz

Mathematical Institute Academy of Sciences of the Czech Republic Žitná 25

11567 Praha 1, Czech Republic

E-mail: opic@math.cas.cz 